\title{
Key rheological properties of hyaluronic acid fillers: from tissue integration to product degradation
}

\author{
Samuel Gavard Molliard1', Jérémie Bon Bétemps', Basste Hadjab, David Topchian², Patrick Micheels², \\ Denis Salomon ${ }^{4}$ \\ 1Research and Development Department, Kylane Laboratoires S.A., Plan-les-Ouates 1228, Switzerland. \\ ${ }^{2}$ Dr. David \& Associates Private Practice, Armadale VIC 3143, Australia. \\ ${ }^{3}$ Dr. Patrick Micheels Private Practice, Genève 1206, Switzerland. \\ ${ }^{4}$ Clinique Internationale de Dermatologie Genève S.A. Private Practice, Genève 1201, Switzerland.
}

Correspondence to: Dr. Samuel Gavard Molliard, Research and Development Department, Kylane Laboratoires S.A., Chemin des Aulx 14, Plan-les-Ouates 1228, Switzerland. E-mail: samuel.gavard@yahoo.fr

\begin{abstract}
How to cite this article: Gavard Molliard S, Bon Bétemps J, Hadjab B, Topchian D, Micheels P, Salomon D. Key rheological properties of hyaluronic acid fillers: from tissue integration to product degradation. Plast Aesthet Res 2018;5:17. http://dx.doi.org/10.20517/2347-9264.2018.10
\end{abstract}

Received: 24 Feb 2018 First Decision: 29 Mar 2018 Revised: 3 Apr 2018 Accepted: 4 Apr 2018 Published: 14 May 2018

Science Editor: Wen-Guo Cui Copy Editor: Guang-Zhe Zhu Production Editor: Cai-Hong Wang

\begin{abstract}
Aim: Over the last 15 years, hyaluronic acid (HA) fillers have become the most popular injectable biomaterial for soft tissue correction. With the increasing number of available HA fillers and the multiplication of facial treatments all over the world, there has been a need from physicians to better understand the HA fillers science. There is especially a growing interest in the science-based evaluation of rheological characteristics which represents an essential tool to guide physicians in the selection of the most appropriate HA fillers, administration techniques and depths of injection for their clinical applications.
\end{abstract}

Methods: Four key rheological parameters (viscosity $\eta$, elasticity $G^{\prime}$, normal force $F_{N}$ and elasticity $E^{\prime}$ ) are measured and discussed on five HA fillers.

Results: These four key rheological parameters are demonstrated to play a pivotal role, in combination with the cohesivity, for better predicting the clinical behavior of HA fillers at different stages of their lifetime.

Conclusion: This article discusses the importance of four key rheological parameters during the main steps of the clinical HA fillers' lifetime, from the product injection to the loss of clinical effects. A better knowledge of these HA fillers' rheological parameters can help the physicians to optimize their aesthetic outcomes, safety and patient satisfaction.

Keywords: Hyaluronic acid fillers, rheological properties, product lifetime, tissue integration, clinical effects 


\section{INTRODUCTION}

Injectable hydrogels known as fillers are commonly used in aesthetic medicine to shape the face or to treat signs of facial ageing, e.g., to smooth superficial wrinkles or to palliate age-related atrophy and ptosis by remodeling some parts of the face. Among these injectable hydrogels, hyaluronic acid (HA) fillers have a dominant and unchallenged position ${ }^{[1]}$. In 2016, according to the American Society for Aesthetic Plastic Surgery (ASAPS), 2.49 million HA filler treatments were performed only in the USA with a high growth of $16.1 \%$ versus the previous year ${ }^{[2]}$. With the rising interest in the HA treatments and the increasing number of available HA fillers on the worldwide market, there is a strong demand from the medical community to better understand the science behind these products in order to optimize aesthetic outcomes and safety. Available HA fillers are designed with different manufacturing technologies ${ }^{[3,4]}$, different HA concentrations ${ }^{[5]}$, different crosslinked three-dimensional network structures ${ }^{[6]}$, different pore size distributions of the fibrous HA networks ${ }^{[6]}$, and different cohesivity levels ${ }^{[7]}$ and rheological properties ${ }^{[8,9]}$. Among the proprietary manufacturing technologies, which all allow obtaining specific rheological properties, we can mention the VYCROSS $^{\text {mo }}$ (Allergan Inc., Irvine, CA, USA), the NASHA ${ }^{\text {tw }}$ (Galderma Pharma S.A., Lausanne, Switzerland) and the $\mathrm{CPM}^{\mathrm{mo}}$ (Merz Pharmaceuticals GmbH, Frankfurt am Main, Germany) technologies. The VYCROSS ${ }^{\mathrm{mm}}$ technology uses a combination of low and high molecular weight of HA during the crosslinking to improve the efficacy of the chemical reaction. The NASHA ${ }^{\text {mi }}$ technology uses a step of controlled particle sizing, after the crosslinking reaction, to obtain specific HA gel textures. The $\mathrm{CPM}^{\mathrm{Tm}}$ technology uses a two-step process during the crosslinking reaction for obtaining a cohesive gel with different crosslinking densities of the HA network. Recently, a novel proprietary manufacturing technology for the production of innovative HA fillers has been discovered. It is the OXIFREE ${ }^{\mathrm{mm}}$ technology (Kylane Laboratoires S.A., Plan-les-Ouates, Switzerland) which is characterized by the extraction of destructive oxygen during the manufacturing process, including during the crosslinking step, for significantly preserving the intrinsic properties of the long molecular weight of HA chains. This new technology provides HA fillers with advanced rheological properties which enable to exhibit strong projection capacities and therefore a high ability to volumize the facial skin tissues. All the fillers available on the market are designed by the manufacturers with the aim to be injected by the physicians into the dermal layer, for the superficial ones or into the subcutaneous tissues, for the products with a higher projection capacity. Due to their clinical applications and the major importance of their mechanical behavior features to achieve safe and good results, the rheological properties of the HA fillers are naturally considered key in the field and it is the reason why many articles have been published on this topic over the past few years ${ }^{[8-12]}$. Some of these articles emphasize the importance of the science-based evaluation of HA fillers and more specifically the rheologic tailoring for guiding physicians to identify the HA fillers that they want to use and to select the most appropriate administration technique and depth of injection ${ }^{[9]}$. Nevertheless, to the best of our knowledge, no published article has evaluated the relevance of the key rheological parameters of a HA filler during its whole clinical lifetime, i.e., from injection of the HA gel into skin tissues to its in vivo degradation over the months, as it is the case in this article. In the light of the assessment of these key rheological parameters, this article also analyzes the mechanical behavior of a novel HA fillers range, benefiting from the OXIFREE ${ }^{\mathrm{ru}}$ technology, in order to better understand the safety and the performance of these new products from the injection into skin tissues up to the loss of the clinical effects. Notably, the projection capacity of these new HA fillers is compared to that of the market leader in the volumizing segment, Juvéderm Voluma ${ }^{\text {ma }}$ (Allergan Inc., Irvine, CA, USA), a device produced according to the VYCROSS ${ }^{\text {mit }}$ technology.

\section{METHODS}

\section{Materials}

Five crosslinked HA fillers intended for facial injection in aesthetic medicine were subjected to flow, oscillatory shear-stress and compression tests with a DHR-1 rheometer (TA Instruments, New Castle, PA, USA). Among these $5 \mathrm{HA}$ fillers, four are manufactured according to the novel OXIFREE ${ }^{\mathrm{m} w}$ technology [Table 1], and one is Juvéderm Voluma ${ }^{\text {mix }}$, manufactured according to the VYCROSS ${ }^{\text {mo }}$ technology. 


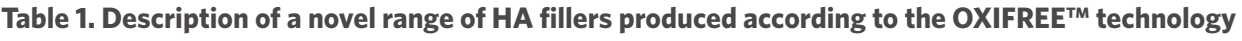

\begin{tabular}{|c|c|c|c|c|}
\hline Product reference & Manufacturer & $\begin{array}{c}\text { Manufacturing } \\
\text { technology }\end{array}$ & $\begin{array}{c}\text { Crosslinked HA } \\
\text { content }(\mathrm{mg} / \mathrm{mL})\end{array}$ & Clinical indications \\
\hline A & $\begin{array}{l}\text { Kylane Laboratoires } \\
\text { SA (Plan-les-Ouates, } \\
\text { Switzerland) }\end{array}$ & OXIFREETM & 15 & $\begin{array}{l}\text { Injection in the dermis or the lips mucosa } \\
\text { Treatment of fine lines, medium-sized skin depressions } \\
\text { of the face area and lips definition or enhancement }\end{array}$ \\
\hline B & & & 18 & $\begin{array}{l}\text { Injection in the deep dermis or the lips mucosa } \\
\text { Treatment of deep skin depressions of the face area and } \\
\text { lips enhancement }\end{array}$ \\
\hline C & & & 21 & $\begin{array}{l}\text { Injection in the fat tissue or into the supraperiostic zone } \\
\text { Restoration of the volume of the face with a high } \\
\text { projection capacity }\end{array}$ \\
\hline D & & & 24 & $\begin{array}{l}\text { Injection in the fat tissue or into the supraperiostic zone } \\
\text { Restoration of the volume of the face with a higher } \\
\text { projection capacity than product C }\end{array}$ \\
\hline
\end{tabular}

\section{Flow test}

The flow test enables to measure the viscosity $\eta$ of the gel. It was performed at a temperature of $25^{\circ} \mathrm{C}$ under shear rate from 0.001 to $1000 \mathrm{~s}^{-1}$ with a cone/plate aluminium geometry of $40 \mathrm{~mm} 2$ degrees and a $50-\mu \mathrm{m}$ gap between the cone and the plate of the rheometer. The value of the viscosity $\eta$ is measured at the shear rate of $1 \mathrm{~s}^{-1}$.

\section{Oscillatory shear stress test}

The oscillatory shear stress test enables to measure the elastic modulus G'. It was performed at a temperature of $25{ }^{\circ} \mathrm{C}$ in shear stress oscillation mode at $1.0 \%$ of strain, within the linear viscoelastic region, with a cone/ plate aluminium geometry of $40 \mathrm{~mm} 2$ degrees and a $50-\mu \mathrm{m}$ gap between the cone and the plate of the rheometer. The measurements were carried out over a frequency range of 0.1-5 Hz. The value of the elastic modulus G' was measured at the physiologically oscillation frequency of $1 \mathrm{~Hz}$.

\section{Compression test in static mode}

The compression test in static mode enables to measure the normal force $\mathrm{F}_{\mathrm{N}}$. It was performed at a temperature of $25{ }^{\circ} \mathrm{C}$ in normal force mode, with a cone/plate aluminium geometry of $40 \mathrm{~mm} 2$ degrees: $1.0 \mathrm{~g}$ of gel was placed between the cone and the plate and the cone was set in contact with the gel and lowered toward the bottom plate, thus compressing the gel. The normal force $\left(\mathrm{F}_{\mathrm{N}}\right)$ was measured for a gap between the cone and the plate of $1.11 \mathrm{~mm}$ (inverse gap $=0.9 \mathrm{~mm}^{-1}$ ).

\section{Compression test in dynamic mode}

The compression test in dynamic mode enables to measure the elastic modulus E'. It was performed at a temperature of $25{ }^{\circ} \mathrm{C}$ in compression oscillation mode at $1.0 \%$ of strain, within the linear viscoelastic region, with a $40-\mathrm{mm}$ plate/plate aluminium geometry and a $0.5-\mathrm{mm}$ gap between the parallel rheometer plates. The measurements were carried out over a frequency range of 0.1-5 Hz. The value of the elastic modulus E' was measured at the physiologically oscillation frequency of $1 \mathrm{~Hz}$.

\section{Data analysis}

All measurements were carried out in triplicate. Data were expressed as the mean \pm standard deviation (SD). Coefficients of variation lower than $10 \%$ were considered as satisfactory. Results were evaluated statistically using Student's $t$-test with a level of significance fixed at $\alpha=0.05$.

\section{RESULTS}

The key rheological properties viscosity $\eta$, static compression $\mathrm{F}_{\mathrm{N}}$, elastic modulus in shear stress $\mathrm{G}^{\prime}$ and elastic modulus in compression E' were measured on the novel range of HA fillers benefiting from the OXIFREE ${ }^{\mathrm{m}}$ technology and on the market leader device Juvéderm Voluma ${ }^{\text {Tx }}$. The results are summarized in Table 2. 
Table 2. Key rheological properties of a novel HA fillers range produced according to the OXIFREE ${ }^{\mathrm{TM}}$ technology and Juvéderm Voluma $^{\mathrm{TM}}$

\begin{tabular}{|c|c|c|c|c|}
\hline Product reference & Viscosity $\eta$ at $1 \mathrm{~s}^{-1}$ (Pa.s) & $\begin{array}{c}\text { Normal force } F_{\mathrm{N}} \text { of } \\
\text { compression at } 0.9 \mathrm{~mm}^{-1}(\mathrm{cN})\end{array}$ & $\begin{array}{c}\text { Elastic modulus } \mathrm{G}^{\prime} \text { in } \\
\text { shear stress at } 1 \mathrm{~Hz}(\mathrm{~Pa})\end{array}$ & $\begin{array}{c}\text { Elastic modulus E' in } \\
\text { compression at } 1 \mathrm{~Hz}(\mathrm{~Pa})\end{array}$ \\
\hline A & $58 \pm 0$ & $12 \pm 1$ & $137 \pm 1$ & $36,080 \pm 1050$ \\
\hline B & $95 \pm 2$ & $21 \pm 2$ & $192 \pm 3$ & $50,130 \pm 1330$ \\
\hline C & $158 \pm 11$ & $43 \pm 4$ & $248 \pm 3$ & $67,021 \pm 1569$ \\
\hline D & $204 \pm 12$ & $71 \pm 7$ & $310 \pm 4$ & $85,765 \pm 1701$ \\
\hline Juvéderm Voluma ${ }^{\mathrm{TM}}$ & $65 \pm 1$ & $15 \pm 2$ & $318 \pm 3$ & $59,000 \pm 1440$ \\
\hline
\end{tabular}

\section{DISCUSSION}

Injectable HA fillers are provided in sterile syringes to the physicians. The HA fillers lifetime begins by the injection of the product into skin tissues and it concludes by the in vivo degradation of the HA biopolymer.

The main steps of the HA fillers lifetime after tissue implantation can therefore be defined as:

Step 1: injection \& integration

This step corresponds to the extrusion of the HA filler through the needle by the physician followed by the in vivo distribution and integration of the $\mathrm{HA}$ gel into the skin, after the injection. Based on the clinical experience, this step is considered to last from few hours to 2 weeks after the injection procedure.

\section{Step 2: projection}

This step corresponds to the phase of skin projection for which the HA filler pull up the tissues thanks to its specific rheological properties. It lasts generally from few months to 2 years, depending on the product formulation, the treated area, the depth of injection and the patient's metabolism.

Step 3: dynamic facial expression

This step corresponds to the phase of facial expression, e.g., when the patient speaks, smiles or eats, for which the gel firmness of the HA filler must be appropriate, i.e., as close as possible of its in vivo environment, to move as one with the skin tissues for offering natural clinical outcomes. This step 3, which is concomitant to step 2, lasts generally from few months to 2 years.

Step 4: degradation

This step corresponds to the in vivo HA filler degradation, with the consequence of the loss of clinical effects. It corresponds to the decrease of the clinical outcomes over time due to the in vivo HA gel degradation in the treated area. The main factors of HA filler degradation are the free radicals, the hyaluronidases, the thermal hydrolysis and the mechanical stress. This step ends generally in the 2 years following the injection procedure.

\section{Step 1 of HA fillers' lifetime: injection \& integration}

Crosslinked hyaluronic acid, even more than the native hyaluronan, is a viscous biomaterial. Nonetheless, manufacturers produce HA fillers which are most of the time considered by physicians as easy to inject through a thin needle of $30 \mathrm{G}$ or $27 \mathrm{G}$. To achieve this challenge, manufacturers develop specific technologies and product formulations to develop HA gels with a low viscosity at high shear rate when they are extruded through a thin needle. The lower this viscosity at high shear rate, the easier is the extrusion force for the physician to push the gel through the needle. Consequently, the viscosity $\eta$ is a key parameter of the injection procedure for a HA filler, affecting its extrusion force.

When the HA gel goes out of the needle, it is distributed into the skin tissues depending on the rheological properties and cohesivity of the gel and the specific physico-mechanical properties of the receiving tissues ${ }^{[4,13]}$. The cohesivity, defined as the capacity of a material not to dissociate, because of the affinity of its molecules 


\begin{tabular}{|l|l|l|}
\hline Gel viscosity & Cohesive gel & Non cohesive gel \\
\hline Gel with low viscosity & & \\
\hline Gel with high viscosity & & \\
\hline
\end{tabular}

Figure 1. Schematic representation of the cohesivity and viscosity of a HA gel. Impact of the cohesivity and viscosity on the shape and behavior of the gel

for each other, is naturally important during the product distribution into the tissues of the treated area ${ }^{[7]}$. However, the rheological properties have also a substantial role at this beginning of the in vivo HA filler lifetime. More specifically, among the rheological properties, the viscosity $\eta$ is the key parameter at this step of the treatment. The viscosity level of the HA filler, combined with its cohesivity profile [Figure 1], define its capacity to remain at the injection site or to spread into the tissues. Thus, a HA gel with a poor viscosity has a higher ability to flow and spread in the tissues in comparison to a gel with a high viscosity. Appropriate viscosity and cohesivity of the HA filler provide the capacity to be easily moldable after injection during massage, allowing the product to be adequately placed, distributed and homogenized within the tissues, without fragmentation of the gel.

The consideration of the viscosity as a key rheological parameter for product distribution, and therefore for tissue integration, is consistent with the clinical uses of the HA fillers by the physicians. Indeed, on the contrary to high viscosity HA fillers, the products with low viscosity are often used to treat superficial indications, where nice and homogeneous tissue integration is especially desired, as for instance for the treatment of fine lines. These products spread easily or quite easily in the skin tissues, sometimes with the implementation of a smooth massage by the physicians, and there are commonly injected in the dermis, sometimes even in the superficial dermis with the "blanching injection technique", without high risk of nodules formation ${ }^{[14]}$.

\section{Step 2 of HA fillers' lifetime: projection}

The normal force $\mathrm{F}_{\mathrm{N}}$ of the HA filler plays a preponderant role during all the implantation phase with an essential effect on the tissues projection capacity ${ }^{[8]}$. The rheological parameter $\mathrm{F}_{\mathrm{N}}$ is defined as the force applied by the gel perpendicularly to its surface when it is compressed. The force $\mathrm{F}_{\mathrm{N}}$ allows the gel to push over the surrounding tissues and to counter the deformation and flattening of the product due to the pressure caused by skin tissues. Therefore, this force reflects the ability of the HA filler to project the skin tissues all along the implantation period. The higher is the normal force $\mathrm{F}_{\mathrm{N}}$ of the filler and higher is the capacity to push the skin tissues to project them [Figure 2].

\section{Step 3 of HA fillers' lifetime: dynamic facial expression}

As a HA filler is commonly administered in the face, it is essential for the patients and the physicians to have a product with a high capacity to smoothly and naturally accompany the mechanical motions of the face due to the dynamic facial expression. In this regard, the dynamic parameters G' (elastic modulus in shear stress) and E' (elastic modulus in compression), representative of the gel firmness in dynamic conditions, are extremely important rheological parameters during this step of the HA filler lifetime ${ }^{[8]}$. The elastic modulus G' represents the ability of the HA filler to resist to dynamic shearing forces (i.e., behavior of the gel for recovering its shape after shear deformation) while the elastic modulus E' represents the ability of the 


\begin{tabular}{|c|c|}
\hline Gel with low $\mathrm{F}_{\mathrm{N}}$ & Gel with high $\mathrm{F}_{\mathrm{N}}$ \\
\hline
\end{tabular}

Figure 2. Schematic representation of the level of normal force $F_{N}$ for a HA gel. Impact on the shape and behavior of the gel for a product with a low $F_{N}$ and a high $F_{N}$
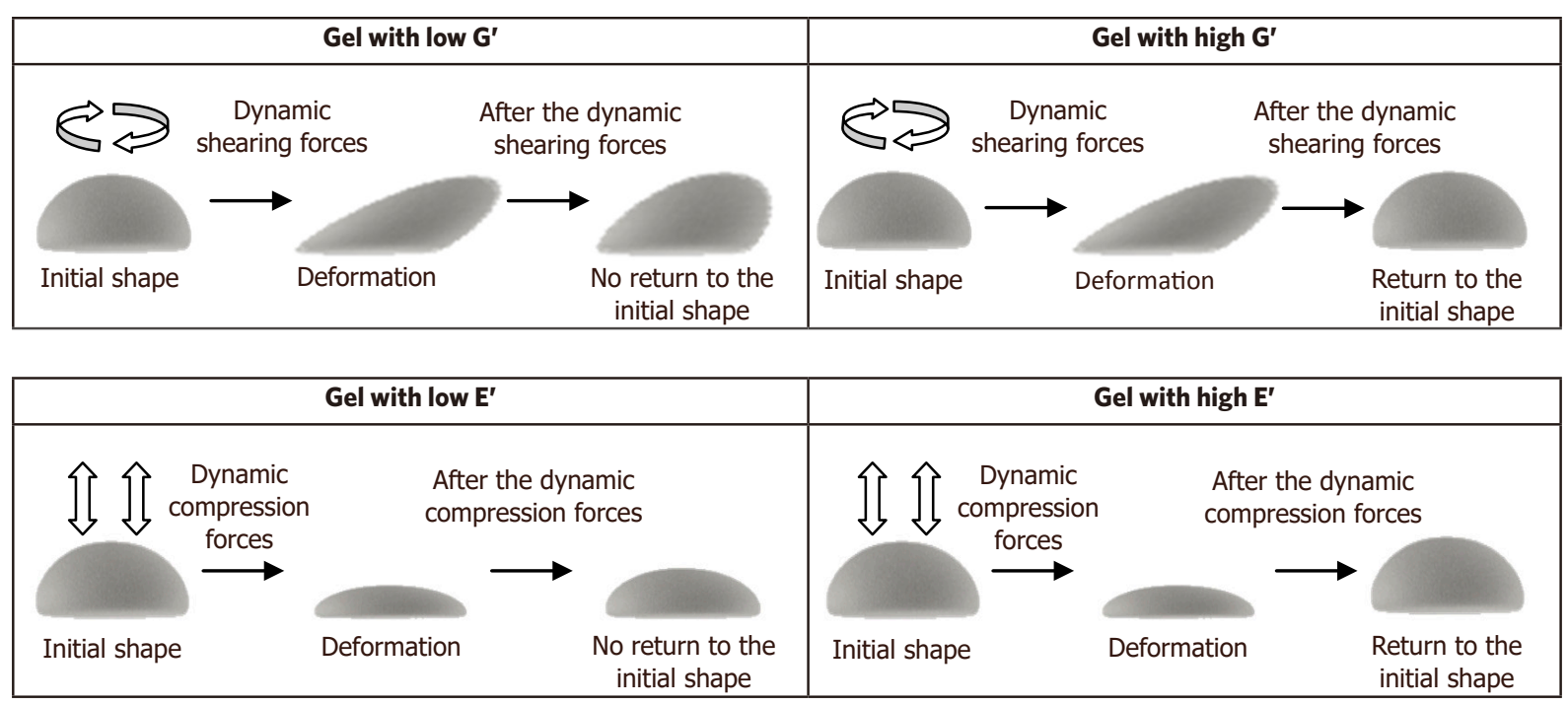

Figure 3. Schematic representation of the level of elasticity $\mathrm{G}^{\prime}$ and $\mathrm{E}^{\prime}$ for a HA gel. Impact of the dynamic shearing and compression forces on the deformation and return to the initial shape of the gel

product to resist to dynamic compression (i.e., behavior of the gel for recovering its shape after compression) [Figure 3]. Because a HA filler is subjected to very high levels of dynamic shear stress but also of dynamic compression stress at each moment of its in vivo life, when for instance, the patient speaks, smiles or eats, the G' and E' properties of the gel implant are key parameters to demonstrate its ability to respond to the mechanical constraints imposed by the dynamic facial expression. Thus, balanced G' and E' dynamic moduli confer a better capacity of the product to well respond to the muscular forces of the skin and to benefit of a better natural effect of the gel implanted in the tissues. Nevertheless, it is important to remember that this natural effect can be fully obtained only if the HA filler has an appropriate position and integration.

\section{Step 4 of HA fillers' lifetime: degradation}

Over time, the HA fillers are degraded by the human body due to the actions of the free radicals, the hyaluronidases, the thermal hydrolysis and the mechanical stress ${ }^{[15]}$. It is important to note that mechanical stress should play a very important role in the loss of the clinical effect. Indeed, as the hyaluronic acid is little by little cut into smaller pieces by the endogenous actors of the skin, the perpetual action of the mechanical expression of the face fosters the loss of implant cohesivity and rheological properties, and especially the normal force $\mathrm{F}_{\mathrm{N}}$ (which enables to maintain the tissues projection) and the viscosity (which enables to resist to flow and spreading). The decrease over time of these key implant properties of the gel is fundamental to understand and explain the progressive disappearance of their clinical efficacy.

\section{Summary}

Following the discussion above, the Table 3 summarizes the key rheological properties for a HA filler, all along its clinical lifetime, from tissue integration to product degradation. 
Table 3. Key rheological properties of HA fillers all along their clinical lifetime. Influence of viscosity $\eta$, normal force $F_{N}$ elastic modulus G', elastic modulus E' and cohesivity at each step of the HA fillers' clinical lifetime

\begin{tabular}{lcccc}
\hline & $\begin{array}{c}\text { Viscosity } \\
\boldsymbol{\eta}\end{array}$ & $\begin{array}{c}\text { Normal force of } \\
\text { compression } \\
\mathbf{F}_{\mathbf{N}}\end{array}$ & $\begin{array}{c}\text { Elastic modulus in Elastic modulus in } \\
\text { shear-stress } \\
\mathbf{G}^{\prime}\end{array}$ & $\begin{array}{c}\text { Cohesivity } \\
\text { compression } \\
\mathbf{E}^{\prime}\end{array}$ \\
\hline $\begin{array}{l}\text { Step 1: injection \& integration } \\
\begin{array}{l}\text { Step 2: projection } \\
\text { Step 3: dynamic facial expression }\end{array}\end{array}$ Major & Major & Major & Major & Major \\
Step 4: degradation & Major & Major & Major \\
\hline
\end{tabular}

\section{Illustration of the key rheological properties with a novel range of HA fillers}

The novel range of HA fillers obtained according to the OXIFREE ${ }^{\mathrm{m}}$ technology was designed with the aim to offer optimized cohesivity and rheological properties for clinical indications covered by each product. Two of these HA fillers (A and B), designed for dermal injection, i.e., superficial administration, have lower viscosities to obtain optimal injection and HA distribution/integration in this skin layer. The normal forces of compression have been selected to deliver an efficient intradermal projection to treat superficial to medium indications of the face, e.g., to treat fine lines or medium to deep-sized depressions of the skin. The 2 other HA fillers of this range (C and D), were designed for subcutaneous injection and to that end, they have high viscosities and high normal forces of compression to procure an optimal capacity of tissues projection in the treatment of the facial contours. Especially, it is notable to observe that product $\mathrm{D}$, intended for the restoration of the volume of the face, has a very strong ability to project the skin tissues thanks to its very high normal force of compression and its high viscosity, even in front of Juvéderm Voluma ${ }^{\mathrm{tw}}$, the worldwide market leader in the segment of the volumizing HA products. Finally, it is important to outline that all HA fillers of this range have both high elastic moduli G' and E', which give a powerful capacity to all the gel implants of this range for withstanding to the mechanical stress in shearing and compression, with the essential purpose to move as one with the skin tissues and therefore to provide natural clinical outcomes, particularly throughout the dynamic expression of the face.

\section{Conclusion}

HA fillers play an increasingly important role in minimally invasive aesthetic procedures and a broad palette of products is now available to the physicians. Science-based evaluation of the HA fillers and especially the analysis of their rheological characteristics was emphasized to be a very useful tool for the physicians to guide them in the selection and usage of the most relevant products, administration techniques and depths of injection for the intended treatments.

The present article highlights the importance of the 4 key rheological properties viscosity $\eta$, elasticy G', normal force $\mathrm{F}_{\mathrm{N}}$ and elasticity $\mathrm{E}^{\prime}$ for better understanding and predicting the behavior of HA fillers during their whole lifetime in the skin tissues, i.e., from their injection in dermal layer or subcutaneous tissues, to their in vivo degradation and therefore, their loss of clinical effects. The purpose of this article is to provide valuable scientific rationale for better explaining the products' behavior during their tissue integration after injection, their capacity to project the skin tissues, their ability to respond to the dynamic facial expression for generating a treatment natural effect, and the gradual disappearance of the clinical benefits.

On the other hand, this article provides a scientific evaluation of a novel range of HA fillers with advanced rheological features with the aim to better predict its clinical behavior. The highlighted findings illustrate how the study of key rheological properties can help the physicians to select the most appropriate product to be administered for the intended use, the volume of product to be injected and the most relevant injection technique to be applied for optimizing aesthetic outcomes, safety and patient satisfaction. 


\section{DECLARATIONS}

\section{Authors' contributions}

Design of the concept and writing of the article: all authors

Obtain all the experimental data: Gavard Molliard S, Bon Bétemps J

\section{Data source and availability}

Experimental data were obtained by Kylane Laboratoires SA

\section{Financial support and sponsorship}

Kylane Laboratoires SA provided the logistical and financial support for the execution of this study.

\section{Conflicts of interest}

Gavard Molliard S is employed by Kylane Laboratories SA, Hadjab B and Bon Bétemps J serve as consultant of Kylane Laboratoires SA.

\section{Patient consent}

Not applicable.

\section{Ethics approval}

Not applicable.

\section{Copyright}

(c) The Author(s) 2018.

\section{REFERENCES}

1. Bui P, Pons Guiraud A, Lepage C. Benefits of volumetric to facial rejuvenation. Part 2: Dermal fillers. Ann Chir Plast Esthet 2017;62:550-9.

2. American Society of Plastic Surgeons. 2016 Plastic Surgery Statistics Report. ASPS; 2016.

3. Micheels P, Sarazin D, Tran C, Salomon D. Effect of different crosslinking technologies on hyaluronic acid behavior: a visual and microscopic study of seven hyaluronic acid gels. J Drugs Dermatol 2016;1:600-6.

4. Tran C, Carraux P, Micheels P, Kaya G, Salomon D. In vivo bio-integration of three hyaluronic acid fillers in human skin: a histological study. Dermatology 2014;228:47-54.

5. Romagnoli M, Belmontesi M. Hyaluronic acid-based fillers: theory and practice. Clin Dermatol 2008;26:123-59.

6. Mondon K, Dadras M, Tillier J, Gavard Molliard S. Influence of the macro- and/or microstructure of cross-linked hyaluronic acid hydrogels on the release of two model drugs. J Glycobiol 2016;5:119.

7. Sundaram H, Rohrich RJ, Liew S, Sattler G, Talarico S, Trévidic P, Gavard Molliard S. Cohesivity of hyaluronic acid fillers: development and clinical implications of a novel assay, pilot validation with a five-point grading scale, and evaluation of six U.S. Food and Drug Administration-approved fillers. Plast Reconstr Surg 2015;136:678-86.

8. Gavard Molliard S, Albert S, Mondon K. Key importance of compression properties in the biophysical characteristics of hyaluronic acid soft-tissues fillers. J Mech Behav Biomed Mater 2016;61:290-8.

9. Sundaram H, Cassuto D. Biophysical characteristics of hyaluronic acid soft-tissue fillers and their relevance to aesthetic applications. Plast Reconstr Surg 2013;132:5S-21S.

10. Sundaram H, Fagien S. Cohesive polydensified matrix hyaluronic acid for fine lines. Plast Reconstr Surg 2015;136:S149-63.

11. Billon R, Hersant B, Meningaud JP. Hyaluronic acid rheology: basics and clinical applications in facial rejuvenation. Ann Chir Plast Esthet 2017;62:261-7.

12. Pierre S, Liew S, Bernardin A. Basics of dermal filler rheology. Dermatol Surg 2015;41:120-6.

13. Micheels P, Besse S, Sarazin D, Quinodoz P, Elias B, Safa M, Vandeputte J. Ultrasound and histologic examination after subcutaneous injection of two volumizing hyaluronic acid fillers: a preliminary study. Plast Reconstr Surg Glob Open 2017;24:e1222.

14. Micheels P, Sarazin D, Besse S, Sundaram H, Flynn TC. A blanching technique for intradermal injection of the hyaluronic acid. Belotero Plast Reconstr Surg 2013;132:S59-68.

15. Stern R, Kogan G, Jedrzejas MJ, Soltés L. The many ways to cleave hyaluronan. Biotechnol Adv 2007;25:537-57. 